\title{
Gestión del cambio y del conocimiento en organizaciones cooperativas y de transformación social: estudio de casos comparados
}

\author{
Sergio García Magariño \\ Universidad Pública de Navarra ${ }^{1}$ \\ sergio.garciam@uninavarra.es \\ Antonio Sánchez-Bayón \\ Universidad Rey Juan Carlos ${ }^{2}$ \\ antonio.sbayon@urjc.es
}

Resumen. Estudio prospectivo sobre los nuevos tipos de organizaciones y su gestión del cambio, así como sobre la transición hacia la sociedad del conocimiento. Se analizan dos propuestas de gestión holocrática que ofrecen factores productivos novedosos, como las tecnologías de aprendizaje y conocimiento y las experiencias de desarrollo de talento $y$ transformación personal y social (propio de organizaciones con sentido $y$ alineadas con Horizonte 2030 y con RSC 3.0). Para ello, se examinan dos casos comparados, en realidades socioculturales diferentes: el Information Systems Costa Rica (ISCR) y el Instituto para el Conocimiento, la Gobernanza y el Desarrollo globales (ICGD) de España. Se pretende sistematizar sus propuestas de organización, operación y resultados, ofreciéndose como pautas de referencia para otras organizaciones orientadas hacia el citado modelo de conocimiento y de transformación social.

Palabras clave: sociología económica; organizaciones de conocimiento y transformación social; talento; tecnologías del aprendizaje y conocimiento.

1 Institute for Advanced Social Research / I-COMMUNITAS; profesor visitante y asesor de la Univ. Nur de Bolivia, e investigador asociado del Instituto de Gobernanza Democrática (Globernance).

2 Prof. Economía Aplicada en URJC, colaborador de Escuelas de Negocios y fellow en LAS-Baylor y GEDePaul. 
Knowledge and Change Management in Social Transformative \& Cooperative Organizations: Comparative Case Study

Abstract. This is a prospective study on the new types of organization and management in the transition towards the knowledge society. Two forms of holacratic management are analyzed and offer innovative productive factors, such as learning and knowledge technologies, as well as experiences of talent development and personal and social transformation (typical of organizations aligned with Horizon 2030 and CSR 3.0). To do so, this paper analyses two study cases, in different socio-cultural settings; namely, Information Systems Costa Rica and the Institute for Global Knowledge, Governance and Development in Spain. The aim is to systematize their proposals regarding organization, operation and results so that they can be made available to other organizations oriented towards the aforementioned model of knowledge and social transformation.

Keywords: sociological economics; knowledge and social transformation corporations; talent; learning E knowledge technologies 


\section{Introducción}

Tras la globalización, puede predicarse que vivimos en mundo en transformación, cada vez más generalizada y acelerada. Se trata de un fenómeno típico de un fin de época: son las crisis de un caduco mundo rígido (dominado por el Estado nación y la gestión burocrática replicantes), dando paso a la emergencia de un mundo flexible, tendente a la aldea global gracias a la gobernanza de las organizaciones internacionales (Andreu et alii, 2019; Sánchez-Bayón et alii, 2018). Como marco de dicha transformación, actualmente nos hallamos en la posglobalización (Sánchez-Bayón, 2019a; Valero et alii, 2018): se trata de un tiempo breve de convergencia mundial, iniciado tras el fin de la globalización (con la crisis de valores de 2008), y vigente hasta el año 2030 (llamado Horizonte 2030). Dicho año resulta clave por ser la fecha de vencimiento de los confluentes programas transformadores de organizaciones internacionales $(v, g$, ODS \& Global Compact-ONU, Future of Work-OIT, Green Deal de UE). Esta convergencia se refiere a la existencia de un plan mundial de actuación que concita al mundo entero, a la humanidad, y de unos períodos particulares que sincronizan la actuación de los países - primero los Objetivos del Milenio y ahora los Objetivos de Desarrollo Sostenible-.

Los Objetivos del Milenio, primero, y los Objetivos de Desarrollo Sostenible, después, han logrado condensar en una misma agenda las máximas aspiraciones que la humanidad ha tenido: erradicar la pobreza, así como sus causas profundas. Esto supone una toma de conciencia colectiva, así como un reto monumental, puesto que lo que se pretende es extremadamente ambicioso. Un elemento vital para lograr los objetivos y metas de desarrollo sostenible será la creación de estructuras organizacionales que operen en diferentes niveles y que permitan encauzar millones de acciones transformadoras de forma colectiva. Por ello, el estudio de organizaciones comprometidas socialmente y que aspiran a incrementar su eficacia mediante la aplicación de marcos y estrategias innovadoras, como las que se examinarán aquí, parece relevante.

Se trata de un punto de no retorno, al producirse la singularidad tecnológica (con la superioridad de AI o inteligencia artificial) y humana (con la normalización transhumanista). Aquellos países y organizaciones que se hayan alineado estarán más cerca de la sociedad del conocimiento y su economía concomitante. Dicho cambio económico ha empezado con la reformulación del paradigma económico-social (vid. figuras 1 y 2), con la transición digital y la transformación de la cultura empresarial y profesional (Sánchez-Bayón, 2020a; 2020b; SánchezBayón y Trincado, 2020). Todo ello ha ido tomando forma en el primer estadio de la economía digital, como es la economía gig o de bolos, que comprende la 
economía colaborativa y circular, más la autónoma y la naranja (Sánchez-Bayón, 2019b; 2019c); actualmente, de cara al Horizonte 2030 y la singularidad, se está avanzando al nuevo estadio, como es la fase wellbeing economics, que combina emprendimiento, talentismo, gestión de la felicidad, RSC 3.0, etc. (González et alii, 2019; Sánchez-Bayón, 2020b). Quienes se queden fuera del proceso de convergencia correrán el riesgo de volverse parte de una sociedad masa de consumo global. En el marco dado, los cambios no son ni buenos ni malos, solo inevitables: en vez de calificarse el mundo como VUCA (acrónimo de volátil, incierto, complejo y acelerado, como estableciera en los años noventa el Ejército estadounidense y popularizara la Universidad de Harvard), pasa a hablarse de un mundo frágil, considerado así por ser de riesgo, líquido, etc., al caducar las instituciones y no cristalizar las nuevas (aumentando la incertidumbre en las interacciones) (Manzanero et alii, 2014; Sánchez-Bayón, 2018); basta con aprender a ser antifrágil (Taleb, 2012), y, finalmente, ágil (Dupont, 2019).

Una acepción coloquial de locura es hacer siempre lo mismo esperando resultados distintos (Sánchez-Bayón, 2020c). Por tanto, debe repensarse todo, revisándose los fundamentos de cada esfera social (derecho, política, economía, etc.), y en especial, aquí, los principios económicos (con sus implicaciones políticojurídicas y sociales). Entre dichos fundamentos (que afectan a las organizaciones económico-sociales), destacan:

- (Re)pensar la economía no solo en términos de renta (PIB), sino de bienestar (incluso, de felicidad, como postularan los clásicos, como Bentham, Stuart Mill, et alii). Se trata de dejar de estudiar la economía mediáticamente (cómo producimos y distribuimos para lograr más riqueza), para hacerlo de manera finalista (por qué producimos y distribuimos; nos proporciona mayor satisfacción y felicidad). En organizaciones así orientadas, cuidarán mucho la claridad y participación en la misión, visión y valores.

- (Re)pensar los recursos y factores de producción distintos a TTK (acrónimo de tierra, trabajo y capital), para prestar mayor atención a TICTAC (tecnologías de la información y comunicación, más las tecnologías del aprendizaje y conocimiento), de modo que tampoco se centre la atención en bienes y servicios, sino en experiencias (orientadas a mejorar satisfacción y bienestar). En tal sentido, una vez más, las organizaciones así orientadas cuidarán en primera instancia la experiencia de sus colaboradores (pues son los primeros clientes y principales embajadores de marca). 
- (Re)pensar los agentes económicos, más allá de las empresas y del Estado (o mejor dicho, sector público), para atender al híbrido de las corporaciones, y no en términos de ficciones jurídicas (como son las personas jurídicas — también conocidas como morales o sociales-), ni de meros sistemas de producción (como lo hace la economía de bienestar estatal), más bien, conviene su consideración como organizaciones sociales donde se lideran personas y proyectos (Andreu et alii, 2019; González et alii, 2019). Por tanto, dichas organizaciones han de ser de pleno sentido, por lo que las interacciones que se den en y desde ellas han de ser conforme a una misión, visión y valores compartidos e integrados. De ahí se llega al compromiso con la RSC 3.0, que permite constatar que las organizaciones poseen pleno sentido y están orientadas a un mayor bienestar (tal como requiere el nuevo estadio de la economía digital, como es la economía de auténtico bienestar o wellbeing economics). De ahí que se hable en la actualidad de empresas sociales (Díaz-Foncea y Marcuello, 2012), cuyo referente ya no son las ONG, sino su versión originaria: las empresas religiosas (Drakopoulou y Seaman, 1998). Los siglos xıx y xx tuvieron sus empresas ideológicas, principalmente, partidos y medios de comunicación, que eran intermediarias del poder. En el s. Xxi, es tiempo de las empresas religiosas, en su sentido etimológico (Sánchez-Bayón, 2018): re-ligare o de estrecha unión con lo espiritual (la divinidad, la ética, etc.) y lo material (la comunidad, la tradición, etc.).

A todo esto se le llama el giro hermenéutico (vid. figura 3) ${ }^{3}$, desde el enfoque GE\&CCM (Escuelas de agua dulce y Escuelas de Negocios, así como en Facultades de Economía con disciplinas duales, tipo economía aplicada, al acoger hoy economía política y principios de economía; Sánchez-Bayón y Trincado, 2020; $2021)^{4}$.

3 Frente al mainstream o pensamiento dominante en economía, que se ha focalizado en lo mediático (cómo se produce y distribuye) y técnico (macroeconometría), o sea, en la medición de la producción nacional (v.g., PIB, PNB), en cambio, hay otras corrientes — cada vez más relevantes - que consideran urgente una reapertura a otras ciencias sociales, que a su vez permitan planteamientos finalistas (por qué se produce y con qué sostenibilidad y nivel de satisfacción), además de humanistas (poniendo en el centro de las relaciones a las personas).

4 Sirva como aclaración sobre GE\&CCM y su giro hermenéutico: comprender la transición digital y la transformación de la cultura empresarial y profesional en curso, que explique mejor la fase de la economía gig (que comprende - como se ha señalado - las manifestaciones de la economía colaborativa y circular, la autónoma y la naranja), y su transición al nuevo estadio, como es wellbeing economics (o economía de auténtico bienestar — no estatal, sino personal y de la humanidad en su conjunto-, que comprende a su vez manifestaciones como el talentismo y la economía de la felicidad), solo ha sido posible desde planteamientos heterodoxos (con la ortodoxia del modelo de economía de bienestar estatal) y praxeológicos (basados en la experiencia de las organizaciones líderes en dichos procesos de transición y transformación, v. g., Global Compact-ONU, Wellbeing Allianze-WEF, GPTW, Merco). Para lograr tal reto es necesario reconectar la economía con el resto de esferas sociales y atender a aspectos más cualitativos, sin dejar de atender a las demandas reales del mercado y los requerimientos de las organizaciones empresariales. De tal suerte, no solo se recupera el espíritu original 
Figura 1. Cambio paradigmático con la transición digital

\begin{tabular}{|c|c|}
\hline VIEJO PARADIGMA (EBE) & $\begin{array}{l}\text { NUEVO PARADIGMA } \\
\text { (EDः de gig a WBE) }\end{array}$ \\
\hline $\begin{array}{c}\text { Economía industrial y material } \\
\text { (modelo mecanicista) }\end{array}$ & $\begin{array}{l}\text { Economía del conocimiento y experiencias } \\
\text { (modelo cuántico) }\end{array}$ \\
\hline Masculino (jerárquico, competitivo) & Femenino (holocrático, comunicativo) \\
\hline Tangible y escaso (fabrica/oficina, bienes) & $\begin{array}{l}\text { Virtual y abundante (móvil, conexiones, ex- } \\
\text { periencias) }\end{array}$ \\
\hline $\begin{array}{l}\text { Cosificación (dinero, horas extra, titulación, } \\
\text { orientación a resultados: medidas higiénicas) }\end{array}$ & $\begin{array}{l}\text { Humanización (bienestar, ocio, talento, orien- } \\
\text { tación a personas: medidas motivacionales) }\end{array}$ \\
\hline $\begin{array}{l}\text { Trabajadores: uniformes, sector secundario, } \\
\text { subordinación (asalariado, heterónomo) }\end{array}$ & $\begin{array}{l}\text { Colaboradores: diversos, sector terciario y } \\
\text { cuaternario, elección (autonomía, responsa- } \\
\text { bilidad) }\end{array}$ \\
\hline $\begin{array}{l}\text { Sistema cerrado: rígido y pobre (burocrático, } \\
\text { para un puesto) }\end{array}$ & $\begin{array}{l}\text { Sistema abierto (autopoiético): flexible y } \\
\text { abundante (creativo y cambiante) }\end{array}$ \\
\hline $\begin{array}{l}\text { Competición (repetición —reparto co- } \\
\text { ciente-, concentración empresarial/multina- } \\
\text { cionales: raíles) }\end{array}$ & $\begin{array}{l}\text { Colaboración (innovación }- \text { modelo West- } \\
\text { minister: } 1 .^{\circ} \text { lo gana todo-, coworking: } \\
\text { mundo elefantes y hormigas) }\end{array}$ \\
\hline $\begin{array}{l}\text { Estudio macroeconómico y econométrico: } \\
\text { principal agente SP }\end{array}$ & $\begin{array}{l}\text { Estudio microeconómico y CCM: principal } \\
\text { agente emprendedor/prosumer }\end{array}$ \\
\hline $\begin{array}{l}\text { Relaciones simples y unidireccionales (B2C), } \\
\text { mononegocio L/P y FPP limitada (costes } \\
\text { fijos) }\end{array}$ & $\begin{array}{l}\text { Relaciones complejas y múltiples (B2C, B2B, } \\
\text { P2P, etc.), multinegocio C/P, FPP variable } \\
\text { (heurística) }\end{array}$ \\
\hline $\begin{array}{l}\text { Átomo (tamaño y localización de oficinas, } \\
\text { stock almacenes, n. }{ }^{\circ} \text { empleados) }\end{array}$ & $\begin{array}{l}\text { Bit (velocidad y ewc, bajo demanda, talento } \\
\text { colaboradores) }\end{array}$ \\
\hline $\begin{array}{l}\text { Manufactura (valor añadido por transfor- } \\
\text { mación de bienes) }\end{array}$ & $\begin{array}{l}\text { Mentefactura (servicio cualificado - con- } \\
\text { cepto/ experiencias-y mayor valor) }\end{array}$ \\
\hline $\begin{array}{l}\text { Gerencia de control (corregir y monopolizar } \\
\text { información) }\end{array}$ & $\begin{array}{l}\text { Gerencia de delegación/coach (reglas y com- } \\
\text { partir información) }\end{array}$ \\
\hline $\begin{array}{l}\text { Resultados por presión y decisiones por temor } \\
\text { (despido) }\end{array}$ & $\begin{array}{l}\text { Resultados por proyectos y logros, y deci- } \\
\text { siones por amor (a lo que hago, con quién, } \\
m-v-v)\end{array}$ \\
\hline
\end{tabular}

Fuente: elaboración propia (Sánchez-Bayón, 2020c; Sánchez-Bayón y Trincado, 2020).

multifocal de la economía política (transferido a principios de economía y, a la postre, a la economía aplicada), sino también de la propia economía, cuando los clásicos ( $v$. g., Bentham, Stuart Mill, Malthus) se cuestionaban la riqueza y bienestar, más allá de la mera medición de la producción nacional, para responder también al nivel de satisfacción ciudadana con el progreso alcanzado (lo que en los códigos JEL viene clasificado como: I31 Bienestar general; Necesidades básicas; Nivel de vida; Calidad de vida; Felicidad). Entre los premios Nobel de Economía referentes en el giro hermenéutico (pasándose de la macroeconometría a la micro-, conectada con otras ciencias sociales), cabe destacar los señalados en la figura 3. 
Figura 2. Cambio paradigmático con la transformación de cultura empresarial

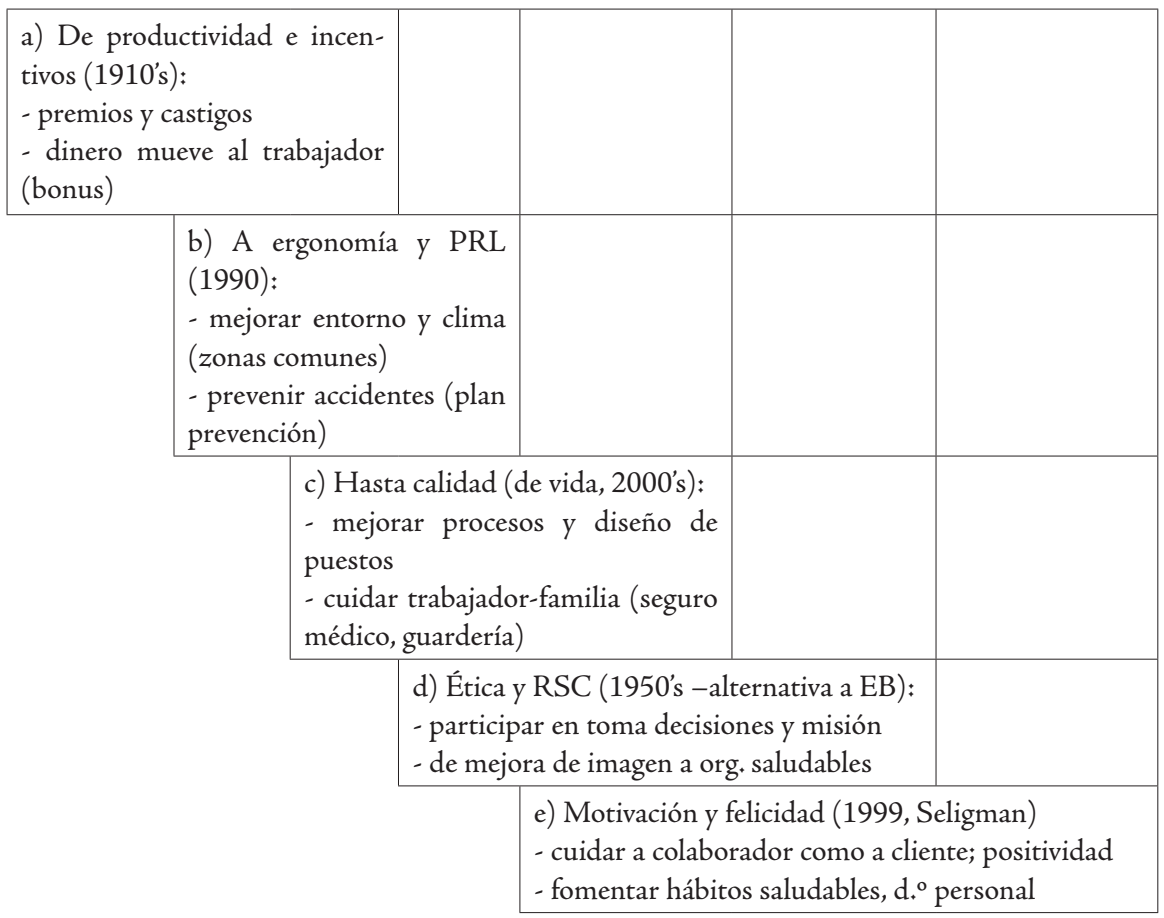

Fuente: elaboración propia (Sánchez-Bayón y Trincado, 2020). 
Figura 3, Giro hermenéutico respaldado por últimos premios Nobel de Economía

Giro hermenéutico:

(Racionalidad limitada y sesgos: aceptación de procesos dinámicos, incertidumbre y error; mix con economía del desarrollo, del comportamiento y de la felicidad:

happiness $\mathcal{E}$ wellbeing economics).

+ Hayk (Esc. Austriaca) y Myrdal: buman action and coop., 1974.

- Simon (econ. comportamiento): bounded rationality, 1978.

- Stigler (econ. información): industrial info and behaviour, 1982.

- Bauchanan (Esc. decisión pública): decision making, 1986.

- Coase (Law E Economics): transaction cost and property rights, 1991.

- Becker (econ. comportamiento-discriminación): buman behaviour, 1992.

- North y Fogel (cliométrica e institucionalismo): institutional change, 1993.

- Nash et alii (Esc. comportamiento y teoría de juegos): game theory, 1994.

- Sen (econ. desarrollo): capacities, development, welfare, 1998.

- Akerlof et alii (econ. comportamiento y felicidad): decision making and life satisfaction, 2002

- Schelling et alii (Esc. comportamiento y teoría de juegos): game theory (conflict), 2005

+ Williamson y Ostrom (econ. organizacional): transaction cost, 2009.

+ Shiller (2013), Deaton (2015), Thaler (2017), et alii

Fuente: elaboración propia (Sánchez-Bayón, 2020a;

Sánchez-Bayón y Trincado, 2020; 2021).

Luego, volviendo a las transformaciones y crisis en curso, los científicos sociales de diferentes disciplinas, así como los filósofos, están de acuerdo con la idea de que estos cambios y estos retos son indicadores de que está teniendo lugar una ruptura histórica y un cambio de época (acelerándose con la crisis de la covid-19). En el corazón de esta coyuntura, se puede percibir un doble proceso: a) de una parte, parece que hay un proceso de desintegración que está erosionando antiguas maneras de pensar, de hacer política, estructuras organizacionales y la vida social y económica; $b$ ) de otra parte, se puede identificar un proceso de integración que parece estar conduciendo al mundo hacia formas más complejas de organización social y de solidaridad y que adoptan al planeta entero como su marco de referencia. Así, estas fuerzas afectan tanto a las mentalidades como a las estructuras de la sociedad, y las organizaciones y empresas también están expuestas a ellas y se ven afectadas. Esta contingencia requiere, de un lado, trascender los modelos tradicionales de organización que son incapaces de navegar por este nuevo escenario, y, de otro lado, encontrar nuevos patrones de actividad 
y de estructuras organizativas que puedan abordar eficazmente los retos mencionados anteriormente. Aunque hay mucho debate teórico sobre las características de estas empresas y organizaciones, parece que esta clase de nuevas estructuras todavía no ha surgido de las bases.

Por tanto, este trabajo se focaliza en organizaciones de conocimiento y transformación social, más exactamente, en la comparativa de dos, ambas de inspiración religiosa ${ }^{5}$, aunque de entornos geográficos y socioculturales diversos, como son a) I.S. Corporación en Costa Rica (ISCR), con más de 40 años de experiencia; b) Instituto para el Conocimiento, Gobernanza y Desarrollo Globales en España (ICGD), con más de un lustro de experiencia. Sendas organizaciones comparten un modelo holocrático, sin jerarquías, sino desde un marco colectivo que guía su trabajo. A fin de abordar el tema sistemáticamente, en primer lugar, se ofrecen algunas reflexiones sobre la naturaleza del debate en el ámbito de la gestión de organizaciones. En segundo lugar, se describen el patrón y los elementos del marco conceptual compartido por un grupo de organizaciones, un marco que parece tener gran relevancia para la modalidad de su acción y que ha dado forma a su trabajo. Por último, se exploran los casos de ISCR y de ICGD, a fin de arrojar luz sobre la praxis de algunos de los rasgos teóricos propuestos en la segunda parte de este documento.

\section{El debate en el ámbito de la gestión y las organizaciones}

El desarrollo del pensamiento en el ámbito de la administración de organizaciones en los últimos tres siglos ha sido riquísimo y no puede ser condensado en unos párrafos. Sin embargo, para iluminar algunas similitudes y diferencias de la propuesta de la sección dos, se hará un esfuerzo por describir ampliamente los contornos del discurso. Por razones analíticas, y conscientes de que son posibles otras muchas clasificaciones, se representan cuatro categorías de teorías: teorías clásicas, teorías del comportamiento, teorías de sistemas y nuevas tendencias. Antes de entrar en debate, no obstante, es preciso hacer una aclaración terminológica.

Una de las características del lenguaje científico es su falta de ambigüedad. Sin embargo, en el ámbito de la administración, las palabras «modelo»y «teoría»se utilizan de dos maneras. Por una parte, se utilizan en sentido «descriptivo». Así, la «teoría»o «modelo» aspira a describir una forma de administración existente. Por otra parte - y este es probablemente el significado más habitual—, «teoría» $\mathrm{y}$ «modelo» adquieren un significado normativo. «Prescriben» cómo deben ser

5 La fe bahaí está focalizada - entre otras cuestiones - en la mejora personal y transformación social, vía capacitación de personas y unión de ciencia y religión. 
manejadas y estructuradas las empresas y las organizaciones para mejorar su rendimiento. Luego, «teoría» y «modelo» se usan indistintamente, aunque, en el lenguaje de las ciencias naturales —un lenguaje aún más preciso-, mientras que las teorías son explicaciones amplias de un fenómeno específico, los modelos son representaciones simplificadas de las dimensiones particulares de tales fenómenos.

Las teorías clásicas incluyen diferentes propuestas sobre organizaciones, pero tienen en común la idea de que las organizaciones deben estructurarse científica y racionalmente, de tal modo que la productividad pueda mejorarse mediante la correcta aplicación de normas claras, canales de comunicación, mecanismos verticales (top-down mechanisms) y jerarquías, y mediante el uso eficiente del tiempo, los procedimientos, la distribución de tareas, la especialización, etc. La analogía utilizada es la máquina y el propósito, más explícito o implícito, es el de aumentar la producción industrial (Gorodiestsky, 1976). Aunque hay diversidad entre los autores, e incluyen a personas como Marx, Smith, Taylor, Ford y Weber, todos ellos parten de la idea de que los datos empíricos deben servir de base para el trabajo de las empresas y las organizaciones (Sánchez-Bayón, 2020b). Los métodos y las técnicas de gestión deben depender de nociones, métodos, tecnologías y procedimientos probados y testados. Esta propuesta mecanicista ha puesto en marcha un debate y una práctica muy dinámicos sobre métodos para gestionar organizaciones de manera más eficiente y eficaz.

Progresivamente, la dimensión humana del trabajo y de las organizaciones ha recibido una mayor atención. ¿Qué tipo de incentivos necesita la gente para ser más productiva? ¿Qué tipo de relaciones humanas deben fomentarse para mejorar el rendimiento de la empresa? Sobre estas cuestiones, también se han llevado a cabo muchas investigaciones y prácticas. Los estudios conductuales realizados principalmente por psicólogos conductistas que consideraban que el interés individual es el motor de la motivación humana- fueron pioneros a este respecto. Así pues, entraron en juego la cuestión de los sueldos y otras formas de estímulos. El campo de la "gestión de recursos humanos» se institucionalizó tanto a nivel empresarial como académico y finalmente se convirtió en un objeto de investigación en sí mismo. Esta tendencia ha evolucionado significativamente hasta ahora y sigue haciéndolo. Se podría decir que la simpatía posmoderna hacia las técnicas de coaching que pretenden recurrir a lo mejor de la gente por el bien de la empresa se sitúan dentro de este segundo grupo de teorías: las humanistas o del comportamiento.

Dentro de este tipo de teorías que se centran en la dimensión humana de las organizaciones, parece haber dos modalidades. En primer lugar, las teorías que solo tratan de mejorar el rendimiento humano pero que no prestan mucha 
atención a la dinámica estructural y organizativa y operan dentro del marco de las teorías clásicas. Además, hay otro tipo de teorías relacionada con lo que se llama «gestión del cambio», «cambio cultural», que se construye sobre el mismo supuesto - que las personas son la clave de una organización-, pero se sigue explorando la dimensión colectiva y evolutiva de la acción humana dentro de las organizaciones (Gaspar, 2011). En cualquier caso, se puede decir que, más allá de la dinámica operativa de las propuestas clásicas, estos modelos subrayan la importancia de las personas para las organizaciones. Las teorías humanistas simbolizan esta preocupación por los seres humanos.

Un tercer conjunto de teorías, que se basan en las anteriores pero que abordan las organizaciones desde un ángulo diferente, podría definirse como teorías sistémicas o funcionales. El nivel de variación dentro de estas teorías es importante. Sin embargo, todas ellas comparten un mismo supuesto subyacente, diferente al de las dos anteriores: las organizaciones son sistemas compuestos por subsistemas autónomos, que cumplen un rol funcional. Bajo este punto de vista, las organizaciones podrían abordarse como un conjunto, como una serie de procesos y componentes interconectados que solo cobran sentido a la luz de la totalidad (Alegre y Galve, 2008). La organización es vista como una entidad compleja integrada en un contexto social y biológico y que evoluciona como respuesta a los cambios de ese entorno. Algunas teorías de sistemas adoptan analogías biológicas para explicar la interconexión de los componentes, procesos y subsistemas de la empresa, así como la conexión entre la organización y el medio ambiente (Félix, 2004), mientras que otras simplemente se adhieren a la noción de sistemas sociales.

Finalmente, un último conjunto de marcos teóricos puede concebirse como corrientes contemporáneas. Este incluye una amplia gama de modelos, algunos de los cuales tienen bases más científicas que otros. Las teorías contingentes que, contrariamente a los modelos funcionales, subrayan la dimensión conflictiva de las relaciones humanas y presentan la gestión organizacional como el arte de gestionar los intereses conflictivos y de resolver pacíficamente tensiones inevitables son un tipo de nueva tendencia. Algunas personas, especialmente como resultado de las nuevas tecnologías de la información y la comunicación, enfatizan en el papel crucial que la tecnología juega en la gestión. El libro Reinventar las organizaciones (Laloux, 2014) expone una indisoluble unión entre la evolución de la conciencia colectiva y el desarrollo de modelos organizacionales y prevé el nacimiento incipiente de organizaciones teal. Otros autores recurren a la vanguardia del conocimiento científico de las ciencias naturales para elaborar enfoques de gestión organizativa. Por ejemplo, enfatizan la necesidad de generar campos en la 
organización para que las personas, a través de la influencia cultural, desempeñen su labor liberando poderes en la autoorganización, de permitir que el caos se traduzca en orden espontáneo una vez se crea una estructura flexible y básica de funcionamiento; en definitiva, de aplicar conocimientos de nuevos paradigmas científicos, como la mecánica cuántica aludida, al ámbito de la gestión (Wheatley, 2006).

Como se señaló al principio, esta breve e incompleta exploración de las teorías organizativas no era un fin en sí mismo ni tampoco pretendía ser exhaustiva. El propósito ha sido, en primer lugar, ilustrar el rico aprendizaje generado por el campo de estudio y práctica de la administración de organizaciones, y, en segundo lugar, allanar el camino para un análisis de un marco nuevo y prometedor que parece generado por los esfuerzos de varias organizaciones que comparten la misma visión.

\section{Un marco emergente}

Después de haber explorado brevemente el debate teórico sobre la gestión organizativa, se prestará atención a un marco conceptual compartido por un grupo de organizaciones que están tratando conscientemente de crear un nuevo patrón de organización social inspirado por la misma visión. Sin embargo, antes de empezar, es necesario mencionar algunos aspectos sobre la relación entre ética y negocio.

Como se ha intentado mostrar, el conocimiento generado sobre la gestión organizativa es vasto. Asimismo, la ética representa un enorme campo filosófico de investigación. Sin embargo, el debate sobre la ética y las organizaciones más allá de las fronteras de la filosofía no es tan fecundo. Por lo general, la gente da por sentado que las empresas éticas son una categoría homogénea. Esta afirmación, no obstante, parece estar lejos de la realidad. Desde cierto ángulo, se puede afirmar que cada empresa se basa en diferentes valores. Por tanto, parece erróneo asumir que los valores son simplemente universales y «buenos». Por ejemplo, algunas empresas valoran el beneficio sobre cualquier otra cosa. Otras empresas valoran el beneficio pero también el medio ambiente. Otras valoran la competencia justa, mientras que otras valoran sus recursos humanos. Para distinguir las diferentes propuestas, se han acuñado diferentes términos. Por ejemplo, la «responsabilidad social de las empresas» es una categoría que se refiere a un marco específico. En este sentido, hay incluso una organización ${ }^{6}$ que ha identificado los principios que deben guiar a las empresas que quieren ser etiquetadas como «socialmente responsables» y promueve este discurso a nivel mundial.

6 Véase <https://www.bsr.org/en/> 
Hay otras modalidades de «empresas éticas» que difieren entre sí. Las «empresas del triple balance», por ejemplo, han ideado un instrumento de contabilidad para ayudar a las empresas y organizaciones a ser a) económicamente, b) socialmente y c) ecológicamente responsables ${ }^{7}$. Desarrollaron una serie de indicadores cuantitativos para supervisar la evolución de la responsabilidad de las organizaciones. La «economía del bien común» es otra tendencia, con su propia teoría, propuesta, indicadores y estrategia de crecimiento para ayudar a las organizaciones a transformarse y ser más democráticas, prósperas y sostenibles. También tienen un decálogo tanto para la administración de empresas como para la economía política (Felber, 2019). El Global Compact de las Naciones Unidas y la Red de Empresas Alternativas y Solidarias (REAS) son otros movimientos dentro de este campo.

Conscientes de la importancia de las estructuras organizativas para encontrar nuevos modelos sostenibles de desarrollo y organización social, así como de la dimensión ética e incluso espiritual de algunos de los problemas que enfrenta la humanidad, un grupo de organizaciones inspiradas en la visión bahaí ${ }^{8}$ de un mundo unificado, pacífico, sostenible y próspero han puesto en marcha un proceso de aprendizaje práctico sobre la naturaleza de las estructuras organizativas, los procedimientos y las prácticas más adecuadas para responder a estos retos. Esta red de organizaciones incluye instituciones tan diversas como la Universidad Nur en Bolivia, la Fundación Badí en Macao, FUNDAEC en Colombia, BASED-UK en el Reino Unido, la Fundación William Mmutle Masetlha en Zambia, el Tahirih Justice Center en EE. UU., CORDE en Camboya, la Asociación Bayán en Honduras o el Instituto de Desarrollo Barli para Mujeres Rurales en la India. De su experiencia ha surgido un marco de acción específico, un marco que evoluciona continuamente y al que pueden y deben contribuir otras organizaciones, como las que se estudiarán en este documento.

Este marco tiene consecuencias para diversas esferas de acción. La más destacada es el desarrollo social y económico. Sin embargo, solo se examinarán aquí las implicaciones directas sobre la gestión organizativa. En la última sección, se

7 Véase <http://www.praxxis.es/es/>

8 La fe bahaí es una religión mundial nacida en Irán en el siglo xix. Su fundador, Baháu'lláh, planteó la necesidad de reorganizar las relaciones humanas bajo la analogía del cuerpo humano, y de crear estructuras sociales que pudieran responder eficazmente a nuestros desafíos globales. Basándose en las tradiciones espirituales de Occidente y de Oriente, la cosmovisión bahaí contiene enseñanzas místicas, filosóficas, teológicas, así como políticas, económicas, prácticas y sociales. Según la Enciclopedia Británica, hay alrededor de siete millones de bahaís en el mundo y la fe bahaí podría considerarse la segunda religión más difundida en el mundo después del cristianismo, en términos de extensión geográfica. Los bahaís del mundo están tratando de involucrar a un número creciente de individuos y organizaciones de ideas afines en un proceso de aprendizaje sobre cómo traducir esos ideales a la realidad. La ciencia y la religión, junto con la experiencia generada en la acción, se consideran las fuentes de conocimiento para tener éxito y avanzar. 
abordarán dos organizaciones que están tratando de aplicar y articular los componentes del marco relacionados con la gestión organizativa.

Existen al menos quince principios que fundamentan las estructuras, procesos y prácticas organizacionales. El primero podría considerarse la perspectiva de «organizaciones orgánicas orientadas hacia el aprendizaje y la transformación social» ${ }^{9}$. La analogía usada para las organizaciones es el cuerpo humano. El cuerpo humano es un ser orgánico, por tanto, las organizaciones deben ver sus componentes como parte de un todo. Se podría decir que las organizaciones tienen ciertas características orgánicas, como el crecimiento, desarrollo, diversificación, integración, o que las partes que asumen funciones y roles temporales que no se corresponden con la programación inicial. Además, las organizaciones tienen sentido en el contexto de su contribución al progreso social. En este sentido, el progreso social exige actuar sobre las personas, las estructuras y las comunidades, así como sobre las relaciones entre ellas, relaciones que deben caracterizarse por la armonía, la unidad y la reciprocidad. Así, las organizaciones están integradas en un contexto más amplio y forman parte de él. La unidad que inspira el orden organizativo no es equivalente a la uniformidad. La analogía del cuerpo humano implica diversidad, descentralización, autonomía de las partes, pero siempre en el contexto de un cuerpo completo. Por muy útil que sea la analogía del cuerpo humano, esta tiene unas limitaciones que deben ser reconocidas para evitar llevar dicha analogía demasiado lejos. Las organizaciones no son literalmente organismos biológicos y sus partes no coinciden con «el cerebro», «las piernas», «los pies». Las organizaciones están compuestas de seres humanos y estos crean relaciones que dan lugar a sistemas, procedimientos, estructuras, valores y una cultura que se pueden estudiar casi independientemente de estos seres humanos.

Una segunda característica que se incluye en la primera declaración es que las organizaciones están orientadas hacia el aprendizaje. Hay abundante literatura acerca de las «organizaciones de aprendizaje» (learning organizations). No obstante, en el marco propuesto, se entiende por aprendizaje una especie de enfoque metodológico que fundamenta el funcionamiento total de la organización, que impregna su cultura y que puede definirse como la interacción dinámica entre cuatro elementos: acción y experimentación constantes; reflexión sobre la acción y la experiencia; estudio e investigación, y consulta o deliberación colectiva. Esta característica también pone de relieve la importancia del conocimiento para el progreso y abre la puerta a un examen más profundo de la naturaleza del conocimiento, sus fuentes y las actitudes correctas hacia él. Basta recordar la idea

9 Para profundizar sobre la concepción bahaí del cambio social, véase García, S. (2015). El Orden Mundial de Baháu'lláh: una aproximación a su propuesta de transformación social desde las ciencias eclesiásticas. Revista Cauriensia, 10, 289-309, en <https://dialnet.unirioja.es/servlet/articulo?codigo=5324106>. 
propuesta anteriormente sobre la influencia que estas organizaciones tienen en el necesario diálogo entre la ciencia y la religión para encontrar nuevos modelos de acción y vida social. Encontrar nuevos patrones para las organizaciones también requiere este constante diálogo entre la ciencia y la religión y entre la teoría y la experiencia. En esta tarea, debe evitarse la fragmentación entre disciplinas y, especialmente, entre teoría y práctica. En concreto, la separación entre teoría y práctica ha llevado a una situación en la que se ha producido una separación artificial entre aquellos que piensan, estudian y elaboran propuestas y aquellos que actúan constantemente de acuerdo con impulsos o bajo órdenes.

Los procesos colectivos de toma de decisiones son otro mecanismo crucial cuya eficacia condiciona el rendimiento de una organización. Hay diferentes modelos: modelos jerárquicos, donde una persona dotada de autoridad decide sobre el resto; modelos asamblearios, donde todos tienen exactamente el mismo derecho a expresarse y las contribuciones están distribuidas equitativamente; modelos de negociación, donde individuos o grupos «interesados» intentan imponer su voluntad a través de medios pacíficos (García y Arjomandi, 2020)... Se ha intentado poner en práctica estos modelos y cada uno tiene sus puntos fuertes y sus limitaciones. Los ágiles no son democráticos y no aprovechan el talento humano. Los democráticos no son eficientes, ya que normalmente se requieren largos períodos de tiempo para tomar decisiones. Las negociaciones favorecen a aquellos con mayor capacidad o poder de negociación. Conscientes de las limitaciones de los actuales procesos de toma de decisiones, autores como Habermas han desarrollado modelos teóricos alternativos. Sin embargo, nunca se han llevado a la práctica. El mecanismo colectivo para llegar a decisiones que proponen estas organizaciones se conoce como «consulta» (García, 2020).

La «consulta» es una exploración colectiva de la realidad que busca generar una visión común y llevar al grupo a decisiones que guíen su acción hacia el bien común. La consulta tiene una dimensión procedimental, así como otra relativa a unos requisitos individuales de los participantes para que esta sea efectiva. Algunos de esos requerimientos para una consulta exitosa incluyen: la necesidad de compartir ideas libremente pero con tacto, evitar ofender al resto y evitar sentirse ofendido; reconocer que las ideas, una vez compartidas, pertenecen al grupo y pueden ser refinadas, rechazadas o aceptadas sin hacer referencia a la persona que las propuso; evitar la insistencia en los argumentos propios; competencia para coordinar la discusión; que el objetivo del interlocutor sea alcanzar ciertos puntos de acuerdo tras un período de tiempo de puesta en común de pensamientos; el aprecio por el valor del consenso y el recurso al voto de la mayoría cuando el consenso no es posible, y, finalmente, la habilidad para trasladar las decisiones 
a la acción colectiva de manera unificada. Este último punto merece una especial atención, dado que muchos de los problemas aparecen en el momento de implementar los acuerdos. La consulta exige que aquellos que tenían puntos de vista diferentes al acuerdo antes de que se adoptara la decisión apoyen sin reservas dicha decisión. De lo contrario, no es posible distinguir entre una buena o una mala decisión. La experiencia, después de un esfuerzo concertado y persistente - todos dando lo mejor de sí mismos-, mostrará si la decisión fue buena o mala ${ }^{10}$.

Un cuarto principio se refiere a la naturaleza del liderazgo. Las organizaciones dentro del marco bajo discusión tratan de aplicar la noción de liderazgo moral o transformador. Este tipo de liderazgo está orientado hacia a) el servicio y b) el empoderamiento y la construcción de capacidad en individuos y grupos, $c$ ) no busca el reconocimiento, así que es «invisible», e) no lo ejercen solamente aquellos en posiciones de autoridad - aunque los que están dotados de autoridad tienen más responsabilidad-, y $f$ ) se esfuerza por fomentar un cambio positivo y cada vez más profundo en la sociedad.

La creación de capacidad también requiere una mención especial. Aunque el liderazgo se centra en el fomento de la capacidad en otros, estas organizaciones tratan de institucionalizar un sistema que eleve la capacidad en todos los niveles, tanto individual como institucional. Esto no solo se manifiesta en la formación constante, sino que impregna toda la cultura de la organización. Por ejemplo, las cosas deben comenzar de manera pequeña, a través de una o dos líneas de acción, y crecer progresivamente, a medida que se adquiere experiencia y se crea capacidad; las personas tienen espacio para desarrollar y utilizar sus talentos, etc.

Otra característica, que se desprende del primer principio, es la importancia del trabajo en equipo, la cooperación y la reciprocidad. Aprovechar los poderes de sinergia y colaboración dentro la organización y entre organizaciones es un área de acción y aprendizaje cada vez más avanzada. Esto incluye el uso y desarrollo del talento humano, la creación de una atmósfera a partir de la cual las potencialidades pueden ser liberadas, la reducción de las brechas de competencia - estricta separación entre los que piensan y los que actúan, o entre los trabajadores manuales y cualificados - y establecer vínculos de colaboración con los sectores público, privado y civil, por mencionar algunas implicaciones.

La justicia y la equidad son otro principio que esas organizaciones tratan de encarnar progresivamente. Hay muchas preguntas en torno a esta cuestiónઃ ¿cuál es la distribución correcta de los salarios para recompensar la experiencia, los re-

10 Para una exploración más completa de las dinámicas de la consulta en el contexto de la buena gobernanza, véase García, S. (2015). Un cuestionamiento de los supuestos que vertebran la sociedad moderna en búsqueda de nuevos modelos de gobernanza: el gobierno, la sociedad civil y la empresa nadando en un mar de complejidad. Cuadernos de gobierno y administración pública, 2 (2), 189, 190, 200-202. 
sultados obtenidos, el nivel necesario de capacitación y la responsabilidad que se asume dentro de las funciones, sin generar excesivas brechas?, ¿cómo pueden utilizarse los recursos de manera más justa?, ¿cómo se puede explorar la realidad de manera más científica para ver las cosas de manera más objetiva?, ¿cómo pueden las relaciones dentro de la organización y con otras personas e instituciones estar libres de prejuicios?, ‘cómo puede impulsarse el progreso de la organización, de una comunidad o de un grupo sin afectar negativamente a otros?

Por último, podría decirse que los tipos de organizaciones que se están debatiendo están tratando de aplicar ciertas ideas relacionadas con la noción de buena gobernanza, pero no siempre se hace referencia explícita a estas características cuando se habla de su marco común. Por ejemplo, la transparencia es importante, pero, junto con la prudencia y la discreción, que a veces son igualmente importantes; la responsabilidad financiera, ecológica y social; la gobernanza corporativa eficaz; mejores canales para la comunicación interna y externa, así como una buena gestión de la comunicación formal e informal; eficiencia, pero un tipo de eficiencia que tiene en cuenta otros valores y que va más allá de la racionalidad instrumental que solo repara en los costos y los beneficios; la necesidad de crear sistemas de inteligencia colectiva que trasciendan la inteligencia individual; la creación de espacios para que diferentes actores, procedentes de diferentes sectores e implicados en temas específicos, se reúnan para hacer diagnósticos compartidos, planificar y actuar juntos; el uso de la tecnología adecuada para aumentar la productividad, pero generando conciencia de los valores que los diferentes dispositivos aportan a la cultura organizativa y a la dinámica colectiva; sensibilidad hacia la cultura y la comunidad local donde se asienta la organización, para evitar cualquier daño involuntario, y devolver a esa comunidad lo que esta le ofrece; seguridad jurídica y normas estables...

Para terminar esta sección y antes de entrar en los estudios de caso, vale la pena mencionar que, a veces, estas organizaciones nombran esta forma de tomar en consideración los principios como «analizar las implicaciones prácticas de los principios espirituales» con vistas a crear «una dinámica coherente entre las dimensiones materiales y espirituales de la existencia». Este es un ejemplo del diálogo entre la ciencia y la religión que consideran necesario para encontrar modelos más eficaces.

\section{Estudios de caso}

En esta sección se examinarán dos organizaciones que están tratando de aplicar el marco descrito anteriormente - una con sede en Madrid (España) y la otra, en San José (Costa Rica) — , a saber : el Instituto para el Conocimiento, la Gober- 
nanza y el Desarrollo globales (ICGD) y Sistemas de Información de Costa Rica (ISCR). Las razones por las que estos dos casos fueron elegidos - en lugar de otros con una trayectoria más larga, como las organizaciones identificadas anteriormente - son varias. ISCR, por ejemplo, está generando un sólido aprendizaje sobre cómo ayudar a las pequeñas empresas a ser financiera, así como ecológicamente, sostenibles. Esto, a pesar de que no ha habido una sistematización formal de la experiencia. Por otra parte, el ICGD es una nueva organización, pero comenzó inspirada en una red de profesores e instituciones establecida hace casi tres años en torno a un máster de la Universidad de Nur, Bolivia, sobre la gestión de organizaciones para el desarrollo. El ICGD ha tratado deliberadamente de aplicar las ideas de esa iniciativa, que comparte el marco señalado anteriormente, tanto a su propio funcionamiento como a la labor de otras organizaciones a las que asesora. De hecho, su misión es explícitamente ayudar a otras organizaciones a este respecto. Una última razón para elegir estas dos organizaciones es la participación directa de los autores en ellas.

\subsection{Instituto para el Conocimiento, la Gobernanza y el Desarrollo globales (ICGD)}

El ICGD fue fundado hace dos años por un grupo de amigos conscientes de la necesidad de contar con estructuras de aprendizaje para el desarrollo local, dedicadas a elevar la capacidad de las personas, los grupos y las instituciones. Los fundadores se encontraban principalmente en España, pero algunos de ellos residían en el extranjero y estaban involucrados en otros proyectos de desarrollo como la Asociación Bayán.

Tras nueve meses de consultas, definieron su misión como «ayudar a organizaciones socialmente comprometidas a aplicar mecanismos de buena gobernanza y a aumentar la eficacia de sus programas». Su visión era, además, crear una organización que reflejara en su propia gestión y en su trabajo las características del marco previamente explorado, y que pudiera contribuir al progreso social en ámbitos relacionados con la educación y la investigación, la gobernanza, el desarrollo y la comunicación. Una vez cristalizada la misión, redactaron un breve documento para recoger las ideas y orientar la acción futura.

El primer desafío fue aplicar la noción de «empezar a pequeña escala, aprender y crecer progresivamente a medida que se construye capacidad». Para ello, de entre tres o cuatro líneas de acción en las que estaban trabajando, decidieron centrarse en una: asesorar a un ayuntamiento (un Gobierno local) para la puesta en marcha de un proceso participativo que permitiera que agentes económicos y organizaciones civiles locales se implicaran en la configuración y ejecución de las 
políticas de desarrollo local de la Concejalía de Desarrollo de Torrelodones. Esta iniciativa recibió la mayor parte de la atención del ICGD durante dos años. De esta manera, pudieron sistematizar la experiencia y definir un programa denominado Laboratorio de aprendizaje colectivo sobre gobernanza y economía, que ha tenido un gran impacto en la vida política, económica y social de la localidad y que está siendo utilizado por la alcaldía de Torrelodones como emblema del pueblo ${ }^{11}$. Actualmente, se están haciendo esfuerzos para ayudar a la Administración local a mantener en marcha el programa por sí misma.

Una vez asentada esta línea de acción, el ICGD emprendió la tarea de elaborar un programa formal de capacitación constituido por cursos que corresponden a cada uno de los aspectos de la buena gobernanza de las organizaciones, tales como la noción de gobernanza organizativa, el análisis y monitorización de los procesos organizacionales de forma holística, el liderazgo, los procesos de toma de decisiones colectivas, la comunicación, el trabajo en equipo, el uso adecuado de la tecnología, las organizaciones orientadas al aprendizaje, y los procesos de desarrollo y cambio social, entre otros ${ }^{12}$. Para ello, además de definir el programa, encontrar los profesores y pensar en la metodología, trataron de encontrar universidades que lo certificaran. Después de explorar muchas modalidades, firmaron un acuerdo con una universidad, CEDEU/URJC. Ahora está en marcha un proceso de aprendizaje sobre cómo promover el programa a un bajo costo. El objetivo es conseguir que un grupo inicial de 15 estudiantes pruebe el programa y así perfeccionar el método utilizado para impartirlo. Se está contactando con varios grupos para ofrecer diferentes opciones. Una vez logrado esto, se prestará atención a pulir los contenidos, con la esperanza de escribir documentos para cada curso.

Del programa Lab surgió orgánicamente una tercera línea de acción en el área de la comunicación. El ICGD tenía una vinculación formal con una empresa de producción audiovisual dirigida por una de las fundadoras. Otro de los fundadores solía colaborar con ella en un programa sobre gobernanza y economía. Sin embargo, habían decidido paralizar ese programa hasta que el Laboratorio (la primera línea de acción) estuviera bien asentado. No obstante, al cabo de un año, uno de los proyectos que surgió del Laboratorio fue un canal de televisión lo-

11 Para un análisis pormenorizado de la naturaleza del impacto del Lab, véanse la página local del Ayuntamiento de Torrelodones (<https://www.torrelodones.es/etiquetas/lab-laboratorio-de-aprendizaje-colectivo >), la noticia sobre el proyecto de salud que surgió del Lab (<https://www.torrelodones.es/images/stories/ noticias/2019/abril/triptico-salud.pdf >), el documento audiovisual de Amaranta.tv (<https://amaranta.tv/ iii-plenaria-del-lab-torrelodones/>), una descripción general en el número 351 (p. 31) de la Revista de Torrelodones de febrero de 2019 (<https://www.torrelodones.es/images/archivos/revista/2019/02_feb19.pdf>), y un caso de éxito, en la p. 43 de la misma revista.

12 Se puede ver una descripción más amplia del máster en su página web: <www.governanzaydesarrollo.org> 
cal. Empresarios que trabajaban en la misma zona decidieron crear un programa dentro de Amaranta.tv (una televisión online que ya existía, dirigida por la misma persona) llamado Torrelodones.tv. El Ayuntamiento apoyó esta iniciativa y pidió al ICGD que organizara para su emisión mesas redondas sobre los temas que abordaba el Laboratorio: gobernanza, economía y desarrollo, medios de comunicación y sociedad... Así, esta línea de acción comenzó a crecer paulatinamente. A medida que comenzó a llegar algo de dinero, aumentaron las oportunidades para involucrar a algunos colaboradores. Por último, el ICGD decidió utilizar el material escrito y audiovisual desarrollado por algunos de sus miembros para proveer contenidos a otra plataforma en línea: www.gobernanza.es.

Además de aplicar esta noción de crear capacidad de forma progresiva y pasar de una línea de acción a varias líneas de acción y de acciones simples a programas más complejos, el ICGD trató desde el principio de establecer un sistema para el aprendizaje, de ir documentando la experiencia y de ir más allá de la realización de proyectos para elaborar programas replicables. A este respecto, el equipo de coordinación reflexiona constantemente sobre sus acciones, estudia iniciativas similares, intercambia documentos, mantiene consultas periódicas, comparte percepciones con otros e intenta sistematizar las experiencias que se consolidan. Este proceso de aprendizaje ya está en marcha, aunque aún podría reforzarse considerablemente. Uno de los retos es trabajar con un grupo de seis o siete personas, con diferentes niveles de implicación y sin apenas personal. Solo hay una mujer a tiempo parcial que reparte su tiempo entre este y otros proyectos. Sin embargo, el círculo de personas que participan en la acción, la reflexión, la consulta, el estudio y la adquisición de conocimientos se está fortaleciendo y ampliando progresivamente.

Asimismo, se están utilizando, en la medida de lo posible, los talentos de los colaboradores que actualmente disponen de menos tiempo. Por ejemplo, hay colaboradores que son muy metódicos y sistemáticos, y están ayudando con la planificación, el presupuesto y la visión estratégica. Otros pueden escribir, por lo que tratan de articular las ideas, plasmándolas en documentos. Esta dinámica requiere generosidad, paciencia, confianza y visión a largo plazo. Una última observación sobre cómo el ICGD trata de aprender colectivamente se refiere a la visión que tiene sobre la diferencia entre proyectos y programas. Los proyectos son iniciativas a corto plazo, que por lo general requieren un nivel elevado de competencia: personas con conocimientos técnicos. Como el ICGD es pequeño, los miembros no pueden asumir muchos proyectos a pesar de las oportunidades. Además, los proyectos no parecen facilitar el aprendizaje colectivo a menos que se haga un esfuerzo extra para documentar los resultados y hallazgos. Por lo 
tanto, el enfoque del ICGD consiste en generar programas, con materiales que puedan ser replicados por otros después de un cierto grado de capacitación. En el caso del programa Lab, todo lo que se ha hecho con empresarios, coordinadores, funcionarios y políticos se ha traducido en borradores de materiales educativos. De este modo, en Andalucía y en las Islas Canarias se está tratando de replicar la experiencia bajo la perspectiva de que el Laboratorio de aprendizaje colectivo sobre gobernanza económica es un programa de dos años después del cual el ayuntamiento se encarga de su gestión con el apoyo de una red de laboratorios. Del mismo modo, el máster que se ofrece constará de contenidos escritos, a fin de que sea lo más independiente posible de los expertos, si bien los profesores del máster requerirán siempre un alto nivel de formación y de experticia en la materia que impartan.

En lo que respecta a los procesos de toma de decisiones, el ICGD trata de aplicar la metodología que se conoce como consulta tanto a su propia labor como a la de otras organizaciones. Esta ha sido una experiencia interesante, ya que se han elaborado materiales formativos, que abordan tanto la mecánica de los procedimientos como los requisitos individuales, para aumentar la capacidad de los coordinadores del Laboratorio de conducir consultas. Ayudar a otras organizaciones a aplicar eficazmente la consulta es un área de aprendizaje prometedora en cuanto a la mejora del rendimiento de las organizaciones.

La cooperación, la reciprocidad y la unidad se manifiestan en la labor del ICGD de diferentes maneras. Por ejemplo, las relaciones entre los miembros aspiran a ser moldeadas por estas fuerzas. Hay conversaciones explícitas sobre este tema cuando ciertas fuerzas como la crítica, la falta de confianza en los demás o las tensiones se introducen en las relaciones. Se liberan poderes y se logra el éxito en función del grado en que se forjan la unidad y la amistad. Asimismo, las relaciones con otras personas y organizaciones siguen también esta línea. Aparte de lo anterior, el funcionamiento del Laboratorio y las dinámicas de las tertulias televisadas sobre gobernanza económica en Amaranta.tv ayudaron al equipo directivo del ICGD a ser más consciente de los poderes liberados por la cooperación. Se prepararon presentaciones, artículos, decálogos y vídeos para explicar las fuerzas que surgen de la cooperación y para describir la manera en que la competencia está impregnando todas las esferas de la actividad humana y obstaculizando la acción colectiva transformadora. Esta vertiente ha despertado especial interés entre las organizaciones más cercanas al ICGD. Finalmente, el ICGD está tratando de contribuir a la cohesión social, ya que considera la fractura social como un riesgo que no suele percibirse hasta que es demasiado tarde. $\mathrm{Su}$ forma de contribuir es, por un lado, concienciar a las organizaciones sobre la 
importancia de que haya verdadera unidad y afecto y sobre el riesgo permanente de tensiones entre individuos y grupos por motivos de género, clase, ideología o nacionalidad, y, por otro, colaborar con otras instituciones que trabajan en el ámbito de la prevención de la radicalización.

Añadir, por último, unas palabras sobre la justicia social, para ilustrar cómo el ICGD trata de alinearse con el marco descrito. En primer lugar, podría decirse que el deseo de contribuir a la justicia social forma parte del mismo ADN del ICGD. Sus fundadores y colaboradores tienen cada uno su propio trabajo, pero dedican mucho tiempo al ICGD a fin de contribuir a la justicia social. Sin embargo, está prevista la contratación de personal a medida que el ICGD disponga de más recursos. El ICGD realiza servicios facturables por sus contratos con los clientes, pero el dinero se utiliza para pagar a los que están directamente involucrados en el proyecto específico en cuestión. El ICGD retiene un porcentaje para perfeccionar la página web, hacer marketing y presupuestar futuros proyectos. Aunque el personal es escaso, en la política salarial y la gestión financiera se tienen en cuenta ciertos criterios: a) los salarios y pagos deben ser dignos; b) la remuneración es mayor o menor según la experiencia, la formación, el tiempo dedicado y el nivel de responsabilidad; c) no hay beneficios individuales, aunque el ICGD trata de ser autosuficiente, sobre todo a través de los precios de las matrículas y otros contratos; d) el dinero no determina la dirección; e) deben reducirse al mínimo los gastos administrativos para invertir más y mejor en los programas; f) la publicidad no puede ser la principal estrategia de financiación de las líneas de acción del ICGD relacionadas con la comunicación.

\subsection{Sistemas de Información Costa Rica (Information Systems Costa Rica - ISCR)}

El siguiente caso es ISCR. Se trata de una iniciativa en Costa Rica para explorar las implicaciones de un marco de «principios y valores universales» para el funcionamiento de una empresa socialmente comprometida.

Desde el inicio del proyecto, ISCR ha pasado por diferentes etapas, dentro de las cuales se han afrontado muchos retos que han requerido decisiones, ajustes y cambios por parte del actual director, desafíos que a continuación se exploran para su análisis y discusión. La tarea no ha sido fácil, pero ha generado muchas lecciones con resultados alentadores hasta ahora. En palabras del director, que contribuyó a sistematizar su experiencia para este documento, «se espera que el análisis de este caso suponga un punto de partida sobre el que desarrollar muchas iniciativas que apliquen con éxito una gama de principios éticos en la gestión de las organizaciones». 
Para ayudar a comprender el contexto en el que se presenta el caso, vale la pena mencionar algunos aspectos relacionados con el mundo de los negocios. Ello permitirá comprender mejor las razones por las que son cada vez más urgentes iniciativas de este tipo. Lo que hace que esta tarea sea particularmente desafiante es que se aleja del modelo tradicional de negocio que busca obtener beneficios, y explora nuevas formas de integrar las dimensiones sociales, económicas y ambientales en un modelo sostenible desde el punto de vista financiero.

Las empresas participan en interacciones complejas con el objetivo de producir bienes y servicios. Sin embargo, los apremiantes desafíos que afronta la humanidad en lo que respecta a la contaminación ambiental, la creciente desigualdad entre ricos y pobres y la incapacidad de los Gobiernos para responder a múltiples necesidades sociales no son sino algunas de las razones por las que se requieren nuevos modelos empresariales.

Desde su fundación, ISCR se dedicó principalmente a la comercialización de productos tecnológicos y contaba al momento de iniciar el proyecto con 85 empleados. Su estructura organizativa consistía en unos seis puestos de dirección que supervisaban varias divisiones operativas. Era común que un directivo fuera responsable de más de diez personas y que las decisiones se tomaran solo al nivel de la dirección, sin la participación de los empleados subordinados.

Como la mayoría de las empresas, la prioridad de ISCR en aquel momento era generar beneficios y sobrevivir en un entorno altamente competitivo. Es importante mencionar que la llegada de internet y de compañías virtuales como Amazon generó múltiples retos, ya que ha permitido adquirir todo tipo de productos a través de la compra en línea y a escala mundial. A esto se suma que en ese momento ISCR estaba atravesando un proceso de cambio generacional al asumir uno de los hijos del fundador la responsabilidad total de la empresa. Según el periódico especializado El Financiero, solo un 30\% de las empresas sobreviven a una segunda generación de gestión (2016).

El ISCR se enfrentó entonces a varios desafíos. En primer lugar, tenía que encontrar la manera de dar continuidad a una empresa que había nacido 35 años antes y que ahora carecía de su fundador. También tenía que encontrar la manera de adaptarse a los constantes cambios tecnológicos y a las situaciones económicas imprevisibles. Además, en la empresa no se abordaban conscientemente las cuestiones relacionadas con la remuneración, el volumen de trabajo, las responsabilidades en cada puesto, la adopción de decisiones, las oportunidades de mejora y el desarrollo de la capacidad. Por otra parte, aunque no se favorecían prácticas que promoviesen la corrupción, tampoco se las rechazaba categóricamente desde una perspectiva ética. Además, las dificultades en las relaciones internas o externas 
se abordaban indirectamente, sin mucho diálogo con los interesados. Era común que algunas situaciones o problemas que requieren de una acción inmediata quedaran completamente ocultos, lo que con el tiempo los empeoraba y los hacía más difíciles de resolver.

En términos operacionales, la organización, al igual que la mayoría de las empresas, se dividió en departamentos, que, aunque relacionados entre sí, se consideraban «islas» que tenían que cumplir sus responsabilidades particulares sin tener en cuenta al resto de la organización. Se generaron y resolvieron planes y decisiones con poca participación. La proyección social era limitada en el sentido de que no había planes o proyectos orientados a la vocación social de la empresa.

ISCR generó la mayor parte de sus ingresos a través de servicios prestados a entidades gubernamentales, tras la obtención de contratos mediante concursos públicos. En el mundo de los negocios, es común que las empresas recurran a pagos ilegales a funcionarios públicos para obtener ventajas y ganar estos concursos, lo que limita enormemente las oportunidades para aquellos que no están dispuestos a emplear estas prácticas. Además, es muy común que las empresas busquen formas de evitar el pago de impuestos reportando beneficios mucho menores. Además, dado que los impuestos de la seguridad social son bastante altos, es habitual tomar medidas para evitar estos pagos al Gobierno, en detrimento del trabajador, que en el futuro verá reducida la cuantía de su pensión.

En cuanto a la cultura corporativa, dentro de la empresa, la competencia limitó las oportunidades de los empleados de crecer y seguir desarrollando sus capacidades. En algunos casos, las personas a cargo temían que otros asumieran sus posiciones si se veía su capacidad. En otras ocasiones, se temía que perderían protagonismo, por lo que evitaban promover a personas que pudieran demostrar mayores capacidades o habilidades.

Dado que los puntos mencionados anteriormente presentaban múltiples desafíos, la alta dirección decidió asumir la tarea de utilizar el ISCR como laboratorio para aprender a aplicar el marco antes mencionado — de valores, enfoques y principios - a la administración de la organización. Una de las estrategias fue transformar el propósito de un objetivo puramente comercial en uno social. Este nuevo modelo de empresa social rompe en muchos aspectos con el modelo tradicional de una empresa dedicada exclusivamente al beneficio, pero se conecta con aquellos otros modelos cuyo sentido de responsabilidad social les hace pensar en las implicaciones ecológicas y sociales de su trabajo.

El primer reto fue cómo encontrar, en una empresa de más de ochenta personas, la forma de introducir nuevas prácticas de gestión basadas en principios y valores. Había que demostrar que una empresa de este tipo no solo podía ser 
económicamente sostenible, sino que también podía generar valor para los accionistas, así como para los empleados y sus familias. Además, dado que toda la empresa se vería afectada de una forma u otra, todos tenían que conocer el nuevo plan de transformación organizacional, sus intenciones y los resultados esperados.

En cuanto al inicio del plan, cuando se inició, la empresa ISCR contaba con 35 años desde su fundación, por lo que un número significativo de empleados había estado trabajando durante más de veinte años. Como era de esperar, durante un tiempo tan largo, las personas desarrollan hábitos, asumen comportamientos que se consideran aceptables, y hacen las cosas de una manera que desarrolla una dinámica particular y que cristaliza en cierto tipo de cultura. Así pues, el proceso de cambio previsto suponía una transformación de la cultura institucional imperante de forma que reflejara mejor determinados principios y valores.

Otra cuestión era el contexto cultural más amplio relacionado con el entorno social y comercial, que se caracterizaba por la corrupción, la manipulación, la búsqueda continua de los propios beneficios, la falta de transparencia y honestidad; rasgos que se habían naturalizado. Sobre este tema, algunas personas consideran que aquellos que son mejores manipulando el sistema a su favor están dotados de «sabiduría empresarial». Además, existía la idea de que todas las empresas debían esforzarse por ser mejores que otras y que, para ello, debían ajustarse a la realidad mencionada anteriormente. De hecho, se consideraba que una empresa totalmente honesta no es económicamente sostenible, sobre todo si mientras tanto todos los demás aplican lo que se consideran las «reglas del juego» normales.

Otro problema era la cuestión de la credibilidad. A los ojos de los participantes, una propuesta que predica el deseo de generar bienestar para todos, aplicando la justicia, la consulta, la equidad, la transparencia y otros principios, puede parecer un tanto ilusa. Por último, si iniciativas como esta surgieran de creencias religiosas, ello podría dar lugar a sospechas de que se trataba de esfuerzos proselitistas. Por ello, se consideró que lo mejor era hablar explícitamente con los empleados de los objetivos y de la visión planteada para la empresa con toda transparencia y sinceridad.

En cuanto a este proceso de compartir la visión y los planes con todos los involucrados, debido a la novedad de esta iniciativa, era importante que todos pudieran conocer su propósito, sus metas y objetivos, sus componentes, así como las acciones que tomar. El hecho de que el alcance de la iniciativa fuera toda la organización hacía que la comunicación y la consulta fueran fundamentales para evitar temores, malentendidos o incluso falsas expectativas. 
Además, como se estaba asumiendo el compromiso de trabajar por un nuevo modelo organizativo basado en la aplicación de principios, se asumió una gran responsabilidad para cumplir lo prometido y para demostrar sinceridad de propósito.

Uno de los retos más importantes era explicar los conceptos y las consecuencias que giraban en torno a cada uno de los principios. Asimismo, la cuestión que naturalmente se planteaba la gente era si sus empleos se verían amenazados o si les afectaría negativamente de alguna manera.

En relación con el proceso de aplicación de los principios a todos los aspectos de la organización, esta funcionaba con planes, estructuras y normas que requerían una profunda revisión. Había importantes diferencias entre la visión de una organización basada en principios y su funcionamiento en ese momento. Aunque era difícil tener una idea clara de cómo sería la organización en el futuro, se pensó que tendría las siguientes características: habría un ambiente basado en la unidad de propósito; la consulta se utilizaría regularmente para todo tipo de cuestiones; la comunicación sería fluida y constante; la organización tendría la capacidad de aprender y la gestión se basaría en la sistematización; todos los participantes tendrían la oportunidad de desarrollar sus aptitudes y la compensación por el trabajo sería más justa; la labor se realizaría en un espíritu de servicio y la organización sería una fuente de bienestar social y económico para todos; las relaciones se basarían en la transparencia y la pureza de la intención, y todo esto debería lograrse mediante el cuidado y la protección del medio ambiente y el cumplimiento de las leyes y reglamentos del país.

Después de seis años desde que el proyecto comenzó, se pueden extraer aprendizajes y perspectivas relevantes para el análisis y la reflexión. En cuanto a la estructura de la organización, a medida que han pasado los años, la tendencia ha sido reducir los niveles de jerarquía y dar más responsabilidad a un número cada vez mayor de personas. El aspecto negativo de esta tendencia es que hace muy difícil justificar posiciones que están perdiendo relevancia bajo la nueva estructura. Podríamos deducir que este es un efecto generado por la creación de mayores oportunidades para desarrollar la capacidad y para la participación. Tener que dejar ir a varias personas ha sido bastante difícil por muchas razones. La primera es la dificultad que enfrenta la persona que pierde su trabajo. La segunda es que se generan rumores sobre la situación financiera de la organización porque se asume que lo que se busca es reducir los gastos. Por esta razón, la norma ha sido compartir la situación de la manera más transparente y directa posible en cada oportunidad presentada. 
En cuanto a la aplicación de los principios en el contexto de la organización, dado que el objetivo es hacer que la organización en su conjunto refleje la aplicación del marco descrito, es importante analizar el papel que juega cada persona. Los principios se manifiestan a través de acciones concretas, se reflejan en actitudes y formas de hacer las cosas, por lo que deben integrarse en la cultura y las acciones de cada persona. En la actualidad, el director considera que alrededor del 15\% de las personas entiende el proceso que se está desarrollando de una manera más orgánica. Estas son las personas que tienen un nivel de compromiso y convicción altos y que conocen con mayor detalle el marco conceptual que inspira sus esfuerzos.

A medida que continúan los esfuerzos, más personas demuestran una actitud y voluntad de servir a los demás. Algunos también expresan su agradecimiento haciendo su trabajo con excelencia y compromiso con los principios. Cada vez es más común ver a personas consultando en grupos para resolver problemas o tomar decisiones. A nivel de la dirección, se celebran consultas constantes sobre cuestiones relacionadas con la remuneración y las condiciones de trabajo. Tal vez el mayor impacto haya sido el tener un pequeño núcleo de personas consultando todo el tiempo sobre el aprendizaje y las maneras de hacer que las diversas líneas de acción presentadas dentro de la organización contribuyan significativamente a la sociedad.

En relación con la construcción de una organización basada en principios que aprende, comparte y contribuye al desarrollo de una nueva civilización, esto requiere nuevas capacidades que actualmente son difíciles de encontrar de manera natural. Existe el desafío de encontrar una manera de generar procesos de aprendizaje que permitan el desarrollo de las capacidades necesarias. Entre otras, algunas de las capacidades identificadas que pueden mencionarse son a) generar planes y aplicarlos eficazmente, b) promover consultas oportunas con la participación de las personas adecuadas, c) actuar con celeridad para resolver situaciones que requieren atención y d) comprender el impacto de las decisiones desde una perspectiva sistémica y orgánica de la organización, e) compartiendo y acompañando el desarrollo de la capacidad en otros.

En general, se considera que nuestros sistemas educativos, desde los niveles básico e intermedio a los niveles avanzados, como la universidad, no preparan adecuadamente a los ciudadanos para afrontar los complejos desafíos de hoy. El mayor reto, entonces, es diseñar o encontrar programas adecuados que permitan el desarrollo de las capacidades mencionadas. En la actualidad, la dirección ha optado por acompañar más estrechamente a las personas clave a fin de transferir 
conocimientos de manera colaborativa para fortalecer las aptitudes, actitudes y capacidades necesarias.

Con respecto a la articulación y la manera de fortalecer los esfuerzos, la experiencia del ISCR hasta ahora parece mostrar que la manera de crecer orgánicamente y desarrollar nuevas oportunidades es compartiendo y aprovechando las capacidades, conocimientos y habilidades de las personas y organizaciones involucradas en el proceso. La aplicación de principios y valores a la empresa en su conjunto le ha permitido aventurarse en nuevas áreas como la tecnología para la educación científica con diversas agencias gubernamentales; la apicultura a través de la participación de apicultores y organizaciones relacionadas; eficiencia energética, y agricultura urbana.

En cuanto al impacto social en cuestión, una de las grandes preguntas, cuando se habla de emprendimiento social, se refiere al impacto real que se está generando. En un nivel básico, el bienestar social se genera cuando las personas trabajan en un entorno armonioso, en condiciones que permiten su desarrollo y estimulan su creatividad, donde reciben una compensación justa por el trabajo que realizan, y donde la salud también es una prioridad. A este nivel, el ISCR cuenta actualmente con un consultorio médico que funciona bajo las normas nacionales de salud y en coordinación con el sistema de seguridad social. Además de los beneficios sociales obtenidos por el cumplimiento de las normas de protección social, la empresa ofrece políticas de gastos médicos complementarios y de por vida. Como medida adicional, en los casos en que los servicios públicos no son eficaces, la empresa interviene y presta asistencia directamente asegurando la atención por medios privados. En materia ambiental, el ISCR cuenta con la certificación ISO 14001-2015, que garantiza el cumplimiento de medidas que protegen el medio ambiente a través de programas de reciclaje, medidas de ahorro energético y uso razonable del agua.

A pesar de lo anterior, podría argumentarse que muchas empresas cumplen todos estos requisitos, por lo que estos esfuerzos no son tan nuevos. Por ello, vale la pena mencionar algunos elementos adicionales que se separan un poco más de los modelos tradicionales. Dentro de la visión del ISCR como empresa social, se han creado tres nuevas entidades dedicadas a diversos temas. Neuroaula promueve la investigación en el uso de la tecnología para la educación científica. Óptimo se dedica a sistematizar y compartir el aprendizaje del ISCR en torno al tema del emprendimiento social. Corona Dorada es la primera empresa productiva basada en un proyecto apícola que busca explorar el impacto económico, social, ambiental y educativo de una empresa productora de miel en un entorno rural. Por último, con el fin de promover y desarrollar estas iniciativas, ISCR re- 
invierte un porcentaje significativo de sus beneficios, lo cual crea una interesante relación entre las dimensiones comercial y social.

Entre sus proyectos más significativos, Corona Dorada está promoviendo una serie de iniciativas destinadas a transformar la actividad apícola y la comercialización de la miel en Costa Rica. En colaboración con apicultores, asociaciones y entidades gubernamentales, se está reforzando la producción, ampliando la cadena de valor y distribuyendo la rentabilidad de manera más justa. Además, se está poniendo mucho empeño en desarrollar productos innovadores basados en la miel, el polen y la cera. También se buscan nuevos socios comerciales en los mercados europeos y asiáticos, donde estos productos puedan valorarse mejor. Un elemento importante que mencionar es que un porcentaje del precio de venta se asignará a proyectos de educación, investigación e impacto social.

Además, con respecto a la sostenibilidad y la proyección de crecimiento, los resultados hasta la fecha han demostrado que la aplicación de principios como la consulta, la unidad, la justicia y la equidad y el cumplimiento de las leyes, entre otros, generan diversos beneficios. Genera sostenibilidad porque la energía se centra en la construcción y la innovación más que en la resolución de conflictos internos y el control de las personas. Asimismo, la organización aprende mediante consultas y puede reaccionar más rápidamente para resolver problemas o situaciones imprevistas. De la misma manera, la transparencia y las relaciones de confianza permiten que los acuerdos comerciales sean justos para todas las partes. El siguiente gráfico, la figura 4, muestra la tendencia de los beneficios durante los últimos nueve años, lo que confirma la sostenibilidad financiera y el crecimiento, a pesar de la regulación ética importante a la que se sometió la empresa.

Figura 4. Beneficios netos de ISCR

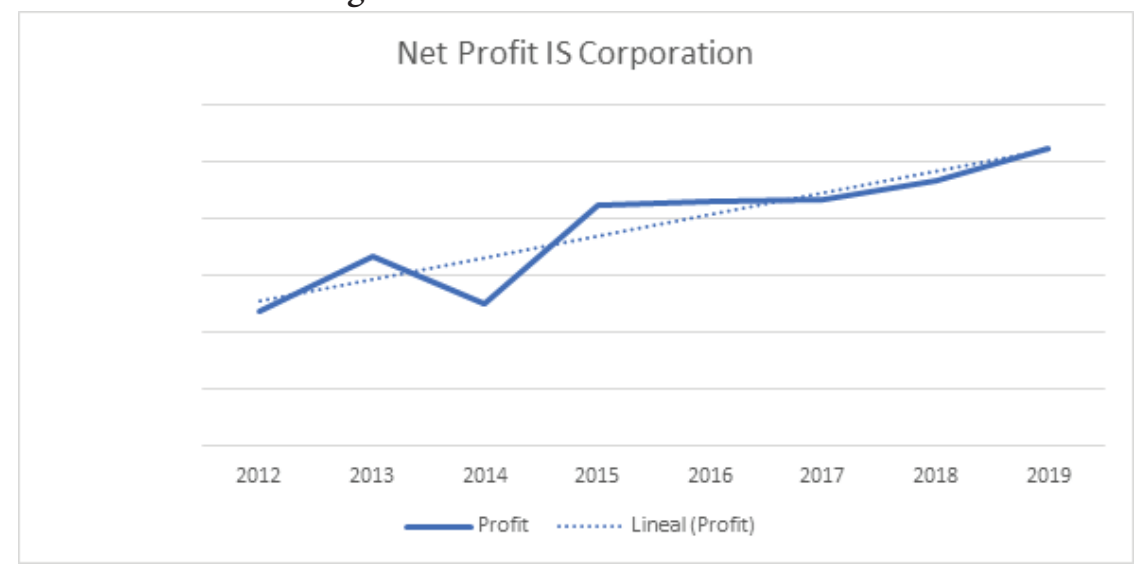

Fuente: elaboración de José Soto, director de ISCR, para este artículo. 
Un elemento crítico para garantizar la continuidad de los esfuerzos ya iniciados por el ISCR es la integración de nuevos participantes que internalizan sistemáticamente todo el concepto y modelo antes mencionados. Con esto en mente, la empresa espera tener una visión más orgánica e integral del proceso de construcción en el que está trabajando. La gerencia del ISCR considera que para que esto suceda es importante compartir el discurso sobre la participación, el desarrollo de capacidades, la generación de bienestar social, económico y ambiental. El lema que el ISCR considera que resume estos conceptos en pocas palabras es «crear valor sin destruir ningún otro valor en el proceso».

Para finalizar, se puede afirmar con bastante seguridad que la experiencia demuestra que un modelo económico basado en la competencia excesiva y la justificación de actos deshonestos para obtener mayores beneficios conduce a relaciones «tóxicas» en las que todos tratan de generar beneficios a expensas de otros. Cuando las empresas se alejan de este modelo y operan bajo supuestos basados en la confianza, la transparencia, la honestidad y la búsqueda del bienestar común, se construyen relaciones justas y duraderas. No parece cierto que una empresa esté obligada a ofrecer los precios más bajos o a infringir las leyes para sobrevivir o explotar a las personas debido a la competencia.

\section{Conclusiones}

Parece existir una necesidad apremiante de nuevas formas de organización que puedan contribuir de manera innovadora a resolver los complejos problemas a los que se enfrenta la humanidad, tales como el cambio climático, la pobreza, los problemas de cohesión social y las desigualdades —entre otros-. Este tipo de problemas requieren la participación de todos los sectores de la sociedad en iniciativas colaborativas que puedan abordar los problemas sociales desde diferentes ángulos.

Además de enfrentarse a problemas polifacéticos, estas nuevas organizaciones se enfrentan a otro reto: operar de tal manera que su funcionamiento alcance mayores niveles de eficiencia y de coherencia. Para ello, diferentes modelos teóricos describen y prescriben nuevas tendencias en el campo de la gestión. Se han explorado cuatro corrientes para ilustrar el rico debate dentro de este ámbito: teorías clásicas, teorías conductuales, teorías de sistemas y nuevas tendencias que van desde los modelos conflictivos a otros modelos que intentan aplicar los conocimientos de la mecánica cuántica y las neurociencias a la gestión organizativa.

En el corazón del documento, se llevó a cabo una exploración de un marco innovador para la gestión que surgió de un conjunto de organizaciones para el desarrollo. Algunas de las características del marco que estas organizaciones aplican a 
su trabajo y a su funcionamiento son las siguientes: conciben a las organizaciones como entidades orgánicas; sitúan el aprendizaje y la generación de conocimientos en el centro de su funcionamiento; utilizan un mecanismo de toma colectiva de decisiones conocido como «consulta»; su concepto de liderazgo gira en torno al empoderamiento y está influido por un espíritu de servicio altruista; tratan de articular las relaciones dentro de la organización y con grupos externos a través de la cooperación y la reciprocidad; una comprensión multidimensional de la justicia informa sus prácticas, y una aplicación adecuada de los múltiples enfoques de la buena gobernanza configura su estructura.

La forma en que entienden su marco va más allá de las propuestas clásicas para conectar la ética con las empresas y las organizaciones. Conceptualizan sus esfuerzos como un intento de crear organizaciones comprometidas con la transformación social, que incorporan conocimientos adquiridos tanto de la ciencia como de la religión, definidos por ellos como dos sistemas superpuestos de conocimiento y práctica que interactúan en lo social.

Por último, se estudiaron dos organizaciones relativamente nuevas - que trabajan bajo el mismo marco innovador mencionado anteriormente-, a fin de poner de relieve, en términos prácticos, su esfuerzo por aplicar conscientemente determinados conceptos, principios y planteamientos surgidos de ese mismo marco para ser más eficaces en su trabajo. Al hacerlo, se destacó - aunque de manera limitada - que el marco tuvo un impacto en los programas emergentes y en el patrón de gestión.

Para concluir, podría decirse que su marco, lejos de limitar su acción, les permite liberar ciertos poderes que emergen de formas sofisticadas de relaciones recíprocas y cooperativas que sirven para impulsar y ampliar el alcance de su labor. Particularmente, en el caso de ISCR — una empresa privada que también genera beneficios sociales - la aplicación de valores y principios contrarios a la competencia prevaleciente por el poder y los recursos no afectó su estabilidad financiera. Por el contrario, el equilibrio económico de la empresa, además de ser positivo, le ha permitido empoderar a los grupos desfavorecidos para generar riqueza propia.

Estas perspectivas extraídas de los casos son preliminares. Seguramente, las organizaciones bajo escrutinio podrían beneficiarse de la experiencia de otras organizaciones que tratan de aplicar otros marcos cercanos al suyo, tales como el de las learning organizations. De hecho, tal como se señaló, el aprendizaje es el modo de funcionamiento principal desde el que operan tanto el ISCR como el ICGD. Desde la óptica del aprendizaje en acción, enfocan el cumplimiento de sus fines sociales, la aplicación de los principios, conceptos y valores del marco que comparten, los intentos de alcanzar la sostenibilidad financiera, etc. A pesar de 
la cercanía, no obstante, se considera que el marco para la gestión que aplican es singular y requiere ser abordado con mayor complejidad tomando otras organizaciones que explícitamente usan ese marco como fuentes de estudio.

En relación con este último punto, cabe señalar algunos estudios ya realizados sobre organizaciones afines al ICGD y el ISCR que pueden suponer un punto de partida para futuras investigaciones. Las tres organizaciones que han alcanzado cierta complejidad en su gestión - mayor que la del ICGD y más profunda que la del ISCR, por los años que llevan trabajando bajo el mismo marco- y que están al alcance de los autores del artículo son la Fundación para la Aplicación y Enseñanza de las Ciencias (FUNDAEC) de Colombia, la Asociación Bayán en Honduras y la Universidad Nur de Bolivia. Sobre todas ellas hay diversos estudios que se centran, principalmente, en sus programas. Por poner un ejemplo, sobre FUNDAEC hay al menos siete tesis doctorales y múltiples libros y artículos científicos que se pueden descargar de su misma página web, fundaec.org. La Asociación Bayán también ha sido objeto de estudio de think tanks importantes como Brookings Institution (2016). No obstante, ninguna de esas publicaciones ha tomado el modelo de gestión de la organización como tema de estudio ni se han colocado en revistas del ámbito de la gestión de organizaciones. Sin embargo, un máster que la Universidad Nur ofrece actualmente en gestión de organizaciones para el desarrollo, en el que uno de los autores de esta publicación colabora como asesor internacional y profesor visitante, puede servir de plataforma para que estudiantes y profesores sigan examinando estas organizaciones y arrojando luz sobre el modelo que se propone - y parece estar funcionando en el prácti$\mathrm{ca}$-. De hecho, el director del ISCR y del ICGD se vieron en Bolivia en el contexto de dicho máster y convinieron una de las entrevistas para este trabajo. El presente artículo, por tanto, no es más que el inicio de una línea de investigación que podría considerarse prometedora. 


\section{Bibliografía}

Alegre, L., Berne, C.y Galve, C. (2008). La empresa. En L. Alegre, C. BerNe y C. Galve (coords.). Fundamentos de economía de la empresa: perspectiva funcional (pp. 9-24). Barcelona: Ariel Economía.

Andreu, A. y Sánchez-Bayón, A. (2019). Claves de Administración y Dirección de Empresas en la Posglobalización. Madrid: Delta Publicaciones.

Drakopoulou, S. y Seaman, P. T. (1998). «Religion and Enterprise: an introductory exploration». Entrepreneurship Theory and Practice, 23 (1), 71-86.

Dupont, L. (2019). «Agile innovation: creating value in uncertain environments». Journal of Innovation Economics \& Management, 28, 1-5.

El Financiero (10 de abril de 2016). «Por qué suelen fracasar las empresas familiares». Disponible en: <https://www.elfinancierocr.com/gerencia/por-quesuelen-fracasar-las-empresas-familiares/2KD4JW6EKVAGDM2CVWE VXW2KEM/story/>

Félix Mateus, A. (2004). «La comunicación en las teorías de las organizaciones. El cruzar del siglo xx y la revolución de las nuevas tecnologías. Una visión histórica». Historia y comunicación social, 19, 195-210.

García, S. (2015). «El Orden Mundial de Bahảu’lláh: una aproximación a su propuesta de transformación social desde las ciencias eclesiásticas». Revista Cauriensia, 10, 289-309.

García, S. (2015). «Un cuestionamiento de los supuestos que vertebran la sociedad moderna en búsqueda de nuevos modelos de gobernanza: el gobierno, la sociedad civil y la empresa nadando en un mar de complejidad». Cuadernos de Gobierno y Administración Pública, 2 (2), 189-202.

García, S. (16 de enero de 2020). «¿Es el consenso algo más que un mantra?». Periodista Digital. Disponible en: <https://www.periodistadigital. $\mathrm{com} /$ politica/opinion/20200116/sergio-garcia-consenso-mantra-noticia-689404243454/>

García, S. y Arjomandi, A. (20 de enero de 2020). «La metafísica del diálogo». El País. Disponible en: <https://elpais.com/elpais/2020/01/15/opinion/1579112865_923448.html>

Gaspar Hernández, H. (2011). «La gestión empresarial, un enfoque del siglo $\mathrm{xx}$, desde las teorías administrativas científica, funcional, burocrática y de relaciones humanas». Escenarios, 9 (1), 38-51.

González, E. y Sánchez-Bayón, A. (2019). Nuevas tendencias en RR. HH. y desarrollo de talento profesional. Porto: Sindéresis.

Gorodiestsky, V. (1976). Introducción al estudio de la organización cientifica del trabajo. La Habana: Editorial Científico Técnica. 
Felber, C. (2019). Change Everything. ZED.

Kwauk, C. y Robinson, J. (2016). Sistema de Aprendizaje Tutorial. Redefining rural secondary education in Latin America. Center for Universal Education of Brookings. Disponible en: <https://www.brookings.edu/wp-content/ uploads/2016/07/FINAL-SAT-Case-Study.pdf>

Laloux, F. (2014), Reinventing organisations. Nelson Parker.

Manzanero, D. (2014). Philosophical challenges of plurality in a global World. Cambridge: Cambridge Scholars Publishing.

Millán Díaz-Foncea, M. y Marcuello, C. (2012). «Las empresas sociales en España: concepto y características». GEZKI, 8, 143-64.

Sánchez-Bayón, A. (2019a). Transición economía gig claves de sociología empresarial y laboral. Encuentros multidisciplinares, 21 (62), 1-19.

Sánchez-BAyón, A. (2019b). «Una historia crítica de sociología del trabajo y de las organizaciones: de «trabajadores de cuello azul y blanco» a «knowmads \& freeriders». Miscelánea Comillas, 77 (151), 431-451. Disponible en: <https://doi.org/10.14422/mis.v77.i151.y2019.008>

SÁnchez-BAyón, A. (2020a). «Renovación del pensamiento económico-empresarial tras la globalización: Talentism \& Happiness Economics». Bajo Palabra, 24, 293-318. Disponible en: <https://doi.org/10.15366/bp2015.10.022>

Sánchez-BAyón, A. (2020b). «Una historia de RR. HH. y su transformación digital: Del fordismo al talentismo y la gestión de la felicidad». Revista de la Asociación Española de Especialistas de Medicina del Trabajo, 29 (3), 198-214.

Sánchez-Bayón, A. (2020c). «Medidas de economía de bienestar que destruyen empleo en la economía digital». Semestre Económico, 23 (55), 87-112. Disponible en: <https://doi.org/10.22395/seec.v23n55a4>

Sánchez-BAyón, A. y Lominchar, J. (2020). «Labour relations development and changes in digital economy: from fragile human resources to agile talentcollaborators»+ Journal of Legal, Ethical and Regulatory Issues, 26 (6), 1-13.

Sánchez-Bayón, A. y Trincado, E. (2020). «Business and labour culture changes in digital paradigm: rise and fall of human resources and the emergence of talent development». Cogito. Multidisciplinary Research Journal, 12 (3), 225-243.

Taleb, N. (2012). Antifragile. New York: Random House.

VAlero, J. y Sánchez-Bayón, A. (2018). Balance de la globalización y teoría social de la posglobalización. Madrid: Dykinson.

Wheatley, M. (2006). Leadership and the new science. San Francisco: BerrettKoehler. 


\section{Webgrafía}

Amranta.tv (2 de octubre de 2019). El LAB de Torrelodones sigue creciendo. Disponible en: <https://amaranta.tv/iii-plenaria-del-lab-torrelodones/> ICGD (web corporativa): <https://gobernanzaydesarrollo.org/ > ISCR (web corporativa): <https://www.iscr.com/website/>

Praxxis Inclusión (web corporativa): <https://praxxis.gal/>

Resilience Business Strategy (web corporativa): <https://www.bsr.org/ en/>

Revista de Torrelodones (feb. 19): <https://www.torrelodones,es/images/archi$\operatorname{vos} /$ revista/2019/02_feb19.pdf>

Revista de Torrelodones (ab. 2019): (<https://www.torrelodones.es/images/stories/noticias/2019/abril/triptico-salud.pdf>

Revista de Torrelodones (jun. 2019): <https://www.torrelodones.es/images/archivos/revista/2019/06_jun19.pdf> 